\title{
SEMANTIC REPRESENTATIONS EXTRACTED FROM LARGE LANGUAGE CORPORA PREDICT HIGH-LEVEL HUMAN JUDGMENT IN SEVEN DIVERSE BEHAVIORAL DOMAINS
}

\author{
Russell Richie* \\ Department of Psychology \\ University of Pennsylvania
}

\author{
Wanling Zou \\ Department of Psychology \\ University of Pennsylvania
}

\author{
Sudeep Bhatia \\ Department of Psychology \\ University of Pennsylvania
}

April 26, 2019

\begin{abstract}
Recent advances in machine learning, combined with the increased availability of large natural language datasets, have made it possible to uncover semantic representations that characterize what people know about and associate with a wide range of objects and concepts. In this paper, we examine the power of word embeddings, a popular approach for uncovering semantic representations, for studying high-level human judgment. Word embeddings are typically applied to linguistic and semantic tasks, however we show that word embeddings can be used to predict complex theoreticallyand practically- relevant human perceptions and evaluations in domains as diverse as social cognition, health behavior, risk perception, organizational behavior, and marketing. By learning mappings from word embeddings directly onto judgment ratings, we outperform a similarity-based baseline and perform favorably compared to common metrics of human inter-rater reliability. Word embeddings are also able to identify the concepts that are most associated with observed perceptions and evaluations, and can thus shed light on the psychological substrates of judgment. Overall, we provide new methods and insights for predicting and understanding high-level human judgment, with important applications across the social and behavioral sciences.
\end{abstract}

\section{Introduction}

Recent advances in machine learning, combined with the increased availability of large natural language datasets, have made it possible to uncover semantic representations that characterize what people know about and associate with a wide range of objects and concepts. In this paper, we examine the power of word embeddings, a popular approach for uncovering semantic representations, for studying high-level human judgment. Word embeddings are typically applied to linguistic and semantic tasks, however we show that word embeddings can be used to predict complex theoreticallyand practically- relevant human perceptions and evaluations in domains as diverse as social cognition, health behavior, risk perception, organizational behavior, and marketing. By learning mappings from word embeddings directly onto judgment ratings, we outperform a similarity-based baseline and perform favorably compared to common metrics of human inter-rater reliability. Word embeddings are also able to identify the concepts that are most associated with observed perceptions and evaluations, and can thus shed light on the psychological substrates of judgment. Overall, we provide new methods and insights for predicting and understanding high-level human judgment, with important applications across the social and behavioral sciences.

* Author contributions: R.R., W.Z., and S.B designed the study, R.R. and W.Z. collected data, R.R. and S.B. performed analyses, R.R., W.Z., and S.B. wrote the paper. Authors declare no conflicts of interest. Correspondence should be addressed to Russell Richie at drrichie@sas. upenn.edu 
People are constantly perceiving, judging and evaluating entities in the world, on the qualities that these entities possess. They may consider, for example, whether a food item is nutritious, whether a political candidate is competent, whether a consumer brand is exciting, or whether the work of an occupation is significant. Such judgments influence every sphere of life, determining the social, professional, consumer, and health outcomes of individuals, as well as the political and economic makeup of our societies. It is thus of critical importance to social and behavioral scientists to develop predictive and explanatory models of human judgment. To have good empirical coverage and practical utility, such models must apply to naturalistic objects and concepts, i.e., the vast range of entities people encounter every day and have rich knowledge about. They should be able to quantify what people know about these entities, and specify how people map this knowledge onto the diverse array of complex judgments they make on a day-to-day basis.

We show how it is possible to quantify knowledge and predict complex judgment with a high degree of accuracy, naturalism, and generality. Our approach relies on word embeddings, a popular class of models in machine learning that use the statistics of word distribution in language to derive high-dimensional vectors for words and phrases (see [1] for reviews). These vectors represent semantic knowledge, so that similar or related words have vectors that are closer to each other in the underlying semantic space. Word embedding-based semantic representations are a useful tool for many practical natural language processing and artificial intelligence applications [2]. However, they also mimic aspects of human semantic cognition, and thus can be used to study how people learn, represent and manipulate the meanings of words [3]. Indeed, word embeddings are able to accurately predict human responses in semantic judgment tasks, such as tasks involving assessments of word similarity, relatedness, and association [4, 5, 6, 7]. They are also useful for modeling other phenomena related to memory, and recent work has used this approach to study priming and lexical access as well as free association, semantic search, and list recall [3, 8, 9, 10, 11]. For this reason, word embeddings are becoming increasingly popular in fields like psycholinguistics, cognitive psychology, and cognitive neuroscience. More recently, scientists have begun to extend word embeddings beyond linguistic and semantic judgment. Many areas of psychology involve associative processing, and measurements of word vector similarity can be used to specify the associations that determine peoples' responses. Based on this insight, researchers have found that word embeddings also predict certain association-based probability judgments, social judgments, and consumer judgments [8, 12, 13, 14].

We find that the structure of knowledge captured by word embedding-based semantic representations can also be applied to study a very wide range of complex human judgments, including judgments that are not easily captured by association-based measures of vector similarity. More specifically, we find that with some training data, it is possible to learn a mapping from word-embeddings space to the judgment domain in consideration, and subsequently make accurate predictions for nearly any entity in that domain. This learnt mapping can also be used to identify the concepts that are most related to the judgment, and thus understand the most important psychological factors for the judgment. To illustrate the broad applicability of this method, we use it to study fourteen types of judgment across seven different domains in the behavioral sciences. These judgments involve naturalistic entities, such as food items, consumer goods, personality traits, job occupations, brands, and public figures, and we examine judgments for these entities on key theoretical dimensions, as identified by prior research.

\section{From representation to judgment}

There are a number of influential methods used by behavioral scientists to quantify representations for the purpose of studying perceptions, evaluations, and other types of judgments for naturalistic entities. These methods can all be considered to be psychometric in that they require human measurements, typically in the form of numeric ratings on scales [15, 16, 17]. Although psychometric techniques have been applied quite successfully across a variety of domains, they typically yield representations of target entity knowledge that are impoverished compared to what people know about the targets. As these approaches rely on explicit participant measurement, is also often costly to collect the information needed to study complex real-world judgment. Of course, it is also impossible to use psychometric techniques to quantify how uncovered representations for judgment targets relate to the hundreds of thousands of other objects and concepts that are involved in the mental lives of individuals and are not necessarily judgment targets.

Thus, a technique is needed which cheaply delivers rich, high-dimensional knowledge representations for a large number of objects and concepts, which can then be used to model judgments. Fortunately, such a technique can be found in word embeddings. As with some psychometric approaches, such as multidimensional scaling [15], word embedding techniques rely on object similarity to uncover entity representations. However, it is not explicit participant similarity ratings or categorization judgments that are used in this analysis, but rather similarity in language, so that pairs of words that occur in similar linguistic contexts are given similar representations. With the recent availability of large-scale natural language online data, as well as new computational resources for analyzing this data, it is possible to use contextual similarity in language to uncover vector representations for all of the words (and corresponding objects and concepts) used in everyday discourse. The dimensions of the vectors are analogous to the latent dimensions uncovered using psychometric methods, but the vectors themselves are typically far richer than what could be obtained 

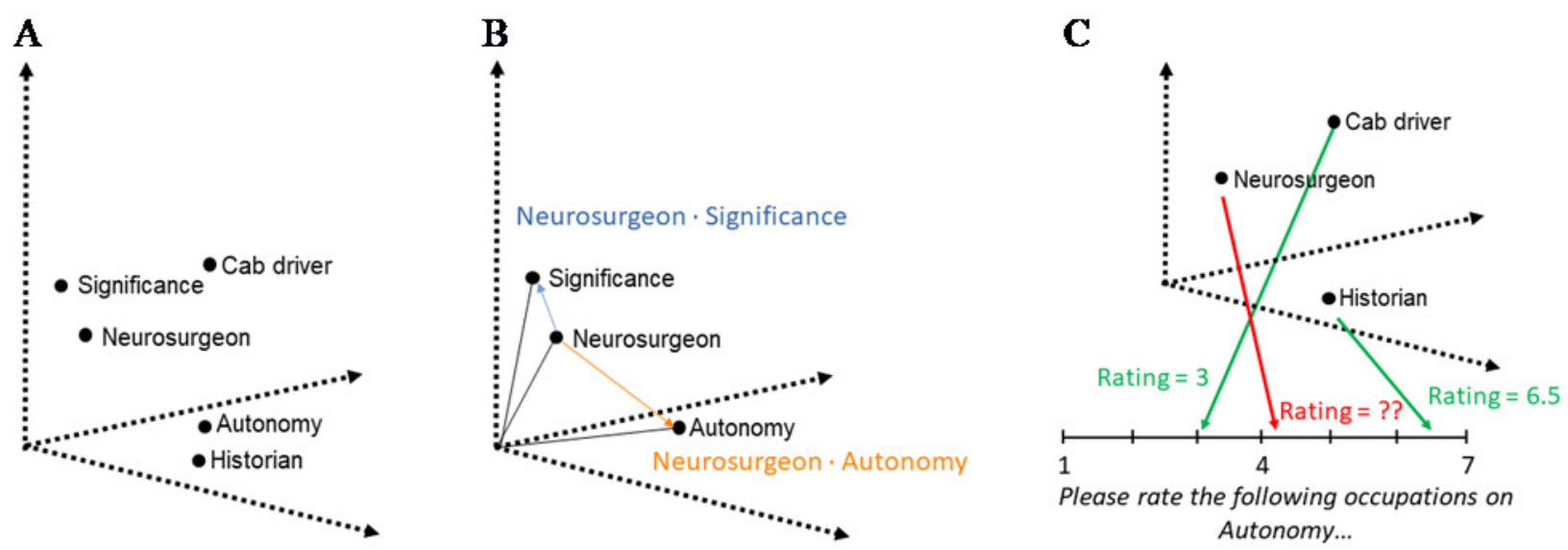

Figure 1: (A) An illustration of a hypothetical three dimensional semantic space with representations for three occupations (neurosurgeon, historian, and cab driver) and two properties (autonomy and significance) on which the occupations are to be judged. (B) An illustration of the vector similarity approach. We use the dot product between the vector for an occupation and the vector for a property to predict the association between the occupation and propery. In this example, neurosurgeon is predicted to be more associated with significance than with autonomy. $(C)$ An illustration of the vector mapping approach. Here we use participant data to learn the mapping from the vector space to the rating dimension in consideration. We train a supervised model to predict autonomy ratings for cab driver and historian based on their vectors, and use the resulting model to predict the autonomy rating for neurosurgeon given its vector.

through survey-based techniques. Most word embedding models have representations for hundreds of thousands of objects and concepts, with each representation involving hundreds of underlying dimensions.

Our proposed approach follows from established psychometric techniques, in which vector representations for entities are used as independent variables in a regression predicting the judgment in consideration. Thus, we also use our word embeddings-based vector representations as inputs into a regression. Due to the relatively high-dimensionality of the word embeddings we use various regularized regression algorithms, and evaluate our model's performance in terms of its ability to predict out-of-sample participant judgments. Intuitively, this approach uses participant judgments for some entities to learn a mapping from the high-dimensional semantic space to the judgment dimension in consideration. When a new entity is presented to be judged, it applies its learned mapping to the semantic vector for the new entity to predict participant judgments for that entity. Some prior work has used this approach to generate word norms in psycholinguistic studies [18], and to study risk perception [19]. In principle, this approach can be applied to any type of human judgment, as long as judgment targets are in the word embeddings vocabulary. It will be successful as long as participant judgment also rely on elements of semantic knowledge represented within the word embeddings space.

We also compare our approach against a baseline model, which involves calculations of vector similarity on the word embeddings space. As similar vectors are semantically related or associated, it is possible to predict the judgment of an entity on a given dimension by calculating how close its vector is to words describing high vs. low ends of the judgment dimension. This measure of relative distance can then be passed through a linear transformation to predict judgments on the same scale as responses elicited from participants. Nearly all applications of word embeddings to predict human semantic and linguistic judgment, memory phenomena such as priming and free association, as well as high-level social judgment and probability judgment, involve calculations of relative vector similarity. Although, this approach performs very well, it is unlikely that it will be successful if the judgments in consideration involves more complex transformations than just simple associations with a set of predetermined judgment-relevant words. That said, our proposed mapping approach can be seen as an extension of the baseline vector similarity approach that uses training data to infer the regions of the semantic space that are most associated with the judgment dimension. As we illustrate below, this property of the mapping approach makes it suitable for inferring, in a bottom-up manner, the psychological substrates of the judgment in consideration.

Figure 1 shows a hypothetical vector semantic space, and illustrates how both our primary (mapping) approach and our baseline (similarity) approach can be applied to representations in this space to predict judgment. Additional details of these two approaches are provided in the methods and materials section. 
Table 1: The judgments of the current study, along with relevant fields, example applications, sample items, and classic references in which these judgments are measured and studied.

\begin{tabular}{|c|c|c|c|c|}
\hline Judgments & Relevant Fields & $\begin{array}{c}\text { Example } \\
\text { Applications }\end{array}$ & Sample Items & $\begin{array}{c}\text { Classic } \\
\text { References }\end{array}$ \\
\hline $\begin{array}{c}\text { Masculinity and } \\
\text { femininity of } \\
\text { traits }\end{array}$ & $\begin{array}{c}\text { Social psychology; } \\
\text { personality } \\
\text { psychology }\end{array}$ & Gender roles & $\begin{array}{l}\text { arrogant, } \\
\text { gentle, } \\
\text { sociable }\end{array}$ & Bem (1974) \\
\hline $\begin{array}{l}\text { Dread-inducement and } \\
\text { unknowability of } \\
\text { potential risk sources }\end{array}$ & $\begin{array}{l}\text { Behavioral economics; } \\
\text { risk analysis; } \\
\text { public policy }\end{array}$ & Risk behaviors & $\begin{array}{l}\text { marijuana, } \\
\text { tsunami, } \\
\text { hackers }\end{array}$ & $\begin{array}{c}\text { Slovic (1987) } \\
{[17]}\end{array}$ \\
\hline $\begin{array}{l}\text { Warmth and } \\
\text { competence of } \\
\text { people }\end{array}$ & $\begin{array}{l}\text { Social psychology; } \\
\text { behavioral } \\
\text { economics }\end{array}$ & $\begin{array}{l}\text { Interpersonal } \\
\text { behavior from dating to } \\
\text { voting }\end{array}$ & $\begin{array}{l}\text { Bill Clinton, } \\
\text { Adolf Hitler, } \\
\text { Mother Teresa }\end{array}$ & $\begin{array}{l}\text { Rosenberg et al. } \\
(1968) ; \text { Fiske et } \\
\text { al. (2002) [22, 23] }\end{array}$ \\
\hline $\begin{array}{l}\text { Taste and } \\
\text { nutrition } \\
\text { of foods }\end{array}$ & $\begin{array}{l}\text { Health psychology; } \\
\text { public health } \\
\text { policy }\end{array}$ & $\begin{array}{l}\text { Dietary behavior; } \\
\text { public health }\end{array}$ & $\begin{array}{l}\text { carrots, } \\
\text { tiramisu, } \\
\text { celeriac }\end{array}$ & $\begin{array}{l}\text { Raghunathan, } \\
\text { Naylor, and } \\
\text { Hoyer (2006) [24 }\end{array}$ \\
\hline $\begin{array}{l}\text { Significance and } \\
\text { autonomy of } \\
\text { occupations }\end{array}$ & $\begin{array}{c}\text { Industrial-organizational } \\
\text { psychology; labor } \\
\text { economics }\end{array}$ & $\begin{array}{l}\text { Career choices; } \\
\text { job satisfaction }\end{array}$ & $\begin{array}{l}\text { cab driver, } \\
\text { neurosurgeon, } \\
\text { historian }\end{array}$ & $\begin{array}{c}\text { Hackman and } \\
\text { Oldham (1976) } \\
{[25]}\end{array}$ \\
\hline $\begin{array}{l}\text { Sincerity and } \\
\text { excitement } \\
\text { of brands }\end{array}$ & $\begin{array}{l}\text { Marketing; consumer } \\
\text { psychology; industrial- } \\
\text { organizational psychology }\end{array}$ & $\begin{array}{c}\text { Purchasing behavior; } \\
\text { organization-public } \\
\text { relations }\end{array}$ & $\begin{array}{l}\text { Home Depot, } \\
\text { Comedy Central, } \\
\text { ING Direct }\end{array}$ & $\begin{array}{c}\text { Aaker (1997) } \\
{[26]}\end{array}$ \\
\hline $\begin{array}{l}\text { Hedonic and } \\
\text { utilitarian value } \\
\text { of goods }\end{array}$ & $\begin{array}{l}\text { Marketing; consumer } \\
\text { psychology; psychology } \\
\text { of motivation }\end{array}$ & $\begin{array}{l}\text { Purchasing and } \\
\text { consumption behavior }\end{array}$ & $\begin{array}{c}\text { chips, } \\
\text { vest, } \\
\text { hammer }\end{array}$ & $\begin{array}{c}\text { Batra and } \\
\text { Ahtola (1990) } \\
{[27]}\end{array}$ \\
\hline
\end{tabular}

\section{Results}

\subsection{Summary of Data}

Our goal in this paper is to evaluate the broad applicability of word embeddings for predicting high-level judgment. Thus, we have chosen seven diverse behavioral domains, spanning a range of disciplines in the behavioral sciences, including social cognition, health behavior, risk perception, organizational behavior, and marketing. For each of the seven domains we have identified two theoretically relevant judgment dimensions, that have been the focus of considerable prior research, and are considered to play a foundational role in human behavior. We have also identified 200 different naturalistic entities for each of these seven domains, selected to cover the range of variation within a domain (see section Item Generation and Table S1 in the Supplementary Information (SI) for details). As we wished to compare the accuracy of our method across these domains, we obtained participant ratings of these entities on the two corresponding judgment dimensions in a single large study. Overall, our study involved 140,000 participant judgments, along 14 judgment dimensions, for nearly 1,400 distinct entities (see SI for all items), spanning seven semantic domains. The judgment dimensions, domains, items, participant instructions, and various implementation details for this study and for the resulting analysis, have been pre-registered at https://osf.io/t7qyb// A summary of judgment dimensions and items we consider, along with example fields and applications and relevant references, is provided in Table 1

In line with our preregistered analysis plan, our main analysis uses a pre-trained word embedding model - word2vec obtained using the skip-gram technique [20], applied to a very large dataset of Google News articles. This space has 300-dimensional vectors for 3 million words and short phrases, and we selected our judgment stimuli to ensure that they were present within the word2vec vocabulary. However we also replicate our basic results with a diverse array of other embedding models trained on various corpora.

\subsection{Predictive Accuracy}

We first evaluated the predictive accuracy of our method for the participant ratings in our data. This initial analysis pertains to predictive accuracy for average participant judgments (i.e. averages of the ratings made on each the fourteen judgment dimensions). As set out in our pre-registration, we tested the ability of a variety of (regularized) regression techniques, across a range of hyperparameters, for mapping pretrained 300-dimensional word embeddings to judgments. We evaluated predictive accuracy in a cross-validation exercise. Our methods and materials section, and Supplementary Information sections Cross-validation and Model Selection and Secondary Models, contain details of this procedure, as well as out-of-sample $R^{2}$ and root mean squared error (RMSE) scores for all model and hyperparameter combinations 

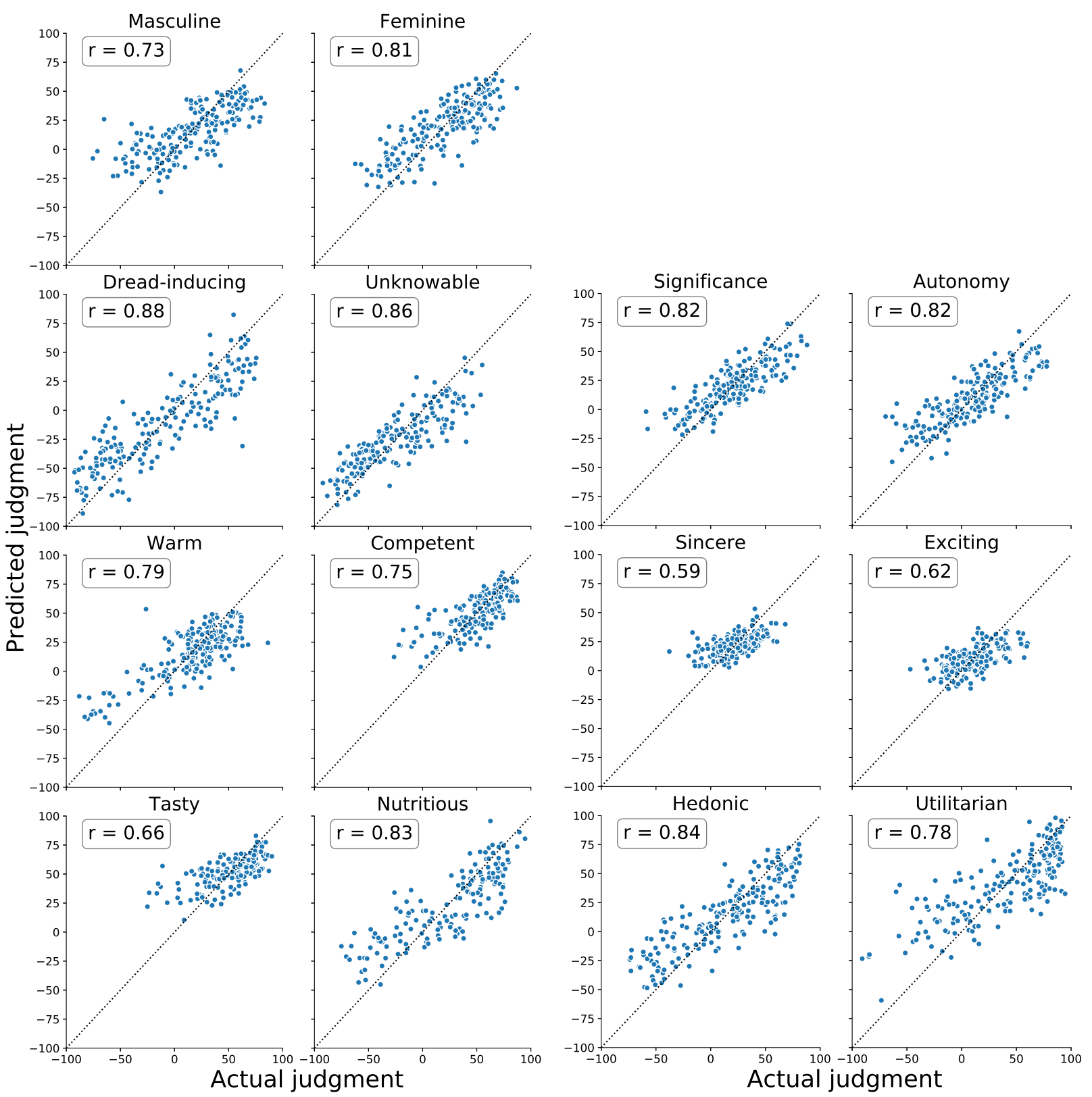

Figure 2: Scatterplots of actual judgments and predicted judgments using leave-one-out cross-validation for each judgment dimension.

that we tested (see Figure S2). A range of models performed well, but we focus here on our best-performing model, a ridge regression with regularization hyperparameter $\lambda$ set to 10 . Figure 2 shows, for each judgment dimension, scatterplots of actual judgments and predicted judgments, along with Pearson correlation coefficients, for this method. Each predicted judgment in the scatterplot was obtained by leave-one-out cross-validation (LOOCV): we trained our ridge regression model on the vectors for all but one judgment target, and then used the trained model to predict the rating for the left-out judgment target based on the target's vector. As can be seen in Figure 2, our approach was able to predict participant judgments with a high degree of accuracy, with an average correlation rate of .77 across the fourteen judgment dimensions, and all fourteen judgments yielding statistically significant positive correlations $\left(\right.$ all $\left.p<10^{-20}\right)$.

We compared the vector mapping approach with the simpler, baseline approach that relies only on the relative similarity of a judgment target to words denoting high vs. low ends of a particular judgment dimension (see Methods and Materials and Supplemental Information Table S2 for more detail). This method also involves fits using leave-one-out cross 
validation, though training the baseline approach only involves learning a linear transformation from the measure of relative vector similarity to the response scale in consideration. We found that the average correlation using this method was .30, which is much lower than that obtained using the vector mapping method. Additionally, the similarity method yields significant correlations only for eleven out of the fourteen tests. Of course, such differences are to be expected, as the mapping method uses the underlying vector space in a much more flexible manner.

We then compared the predictive accuracy of both methods with human inter-rater reliability, as human inter-rater reliability is often thought to place an upper bound on machine performance [5, 28]. For comparison to models predicting mean judgment ratings, we computed reliability two ways. First, we computed the inter-subject correlation (IS-r, [28]), which is the average correlation between one participant's ratings and the average of the rest. This is a commonly used metric in assessing word embeddings' ability to model semantic judgments (e.g., [28]) and is sometimes taken to place an upper bound on machine performance [29]. This correlation came out to 0.60 , whereas our main model surpassed this with an average correlation of 0.77 across judgments, meaning that our mapping method predicts mean human judgments better than do individual human judgments. However, given that our main model is predicting an average judgment rating with word embeddings that more or less constitute the 'average' of human knowledge reflected in word use, it may be more sensible to compare our models' performance to split-half reliability, or the correlation between the average of half the participants with the average of the other half of the participants. Thus, for each judgment dimension, we split participants into two sets, averaged judgment ratings within each set, computed the correlation between the averages, and repeated this process 100 times. The resulting split-half reliability in our judgments averaged across all judgment dimensions is .88, ranging from .69 for taste judgments to .97 for dread-inducing judgments. Figure $3 \mathrm{~A}$ has similarity and mapping method predicted vs actual correlations under leave-one-out cross-validation, inter-subject correlations, and split-half reliabilities for every judgment dimension.

In addition to illustrating the accuracy of our proposed method relative to the baseline method and inter-rater reliability, Figures $3 \mathrm{~A}$ also allows for a comparison of accuracy across judgment dimensions. Such a clean analysis of the differences across domains is possible since we randomly assigned subjects to judgment domains. As can be seen, while performance is good, there is a fair amount of variability in performance across dimensions: while risk source unknowability was predicted with a correlation of .86, food item taste was predicted with a correlation of .66. This variability is likely due to a variety of factors. For example, different judgments may vary in the extent to which they rely on knowledge reflected in language and thus word embeddings. Thus, for example, judgment-relevant attributes for taste may not be represented in our word embedding space. If this were the case, we would expect the accuracy of the baseline similarity approach (which also relies on the word embeddings space) to correlate with the accuracy of the proposed mapping approach. However, the relationship between the two approaches is very mild $(r=.37, p=.19)$. One might also speculate that our judgment dimensions varied in their subjectivity: perhaps taste judgments are merely more subjective than other dimensions. Indeed, as Figure $3 \mathrm{~A}$ shows, mapping model performance is strongly correlated with split-half reliability $\left(r=.88, p<10^{-4}\right)$, such that judgment domains where human raters disagreed more - taste and brand perceptions - were also more difficult for our model. In SI section "Comparing information use across judgment domains" and SI Figure S3, we further compare judgment dimensions, particularly how different judgment dimensions make use of the embeddings in similar and different ways. Interesting cross-domain connections arise, e.g., the similarity in embedding use between autonomy and hedonic value judgments, possibly due to a link between pleasure/happiness and self-determination.

Note that our approach can also be applied to individual-level judgments, thereby accommodating participant heterogeneity. As shown in the SI section Individual-level Modeling, we obtain average correlations of .52 for predicted vs. observed judgments, for the individual participants in each of our fourteen tests. These accuracy rates are lower than those obtained on the aggregate level, likely due to the fact that averaging participant ratings reduces variability in data. However, they are higher than accuracy rates obtained by applying the baseline approach to individual-level judgments, which generates average correlations of .21. As the baseline approach uses the same distances on the semantic space, for all participants, it cannot substantively accommodate participant heterogeneity (though this approach does allow for different participants to map vector similarities onto responses in different ways). To assess the individual-level models relative to inter-rater reliability, we again computed reliability two ways. First, we computed the average pairwise correlation between raters [5]. This correlation came out to 0.34 , whereas our individual-level model predictions correlated with actual judgments at an average correlation of 0.53 . We can also compare individual-level model accuracy with IS-r rates, since IS-r reflects the ability to predict an individual judgment from the mean of other judgments. As stated above, mean IS-r was .60, somewhat above our average individual-level model accuracy of .53. Figure S4 in the SI contains a pointplot like Figure $3 \mathrm{~A}$ with these two measures of inter-rater reliability along with individual-level mapping and similarity models. Overall, for both average- and individual-level judgments, our model performs favorably in comparison to human inter-rater reliability, either exceeding inter-rater reliability or approaching it, depending on choice of inter-rater reliability metric. 


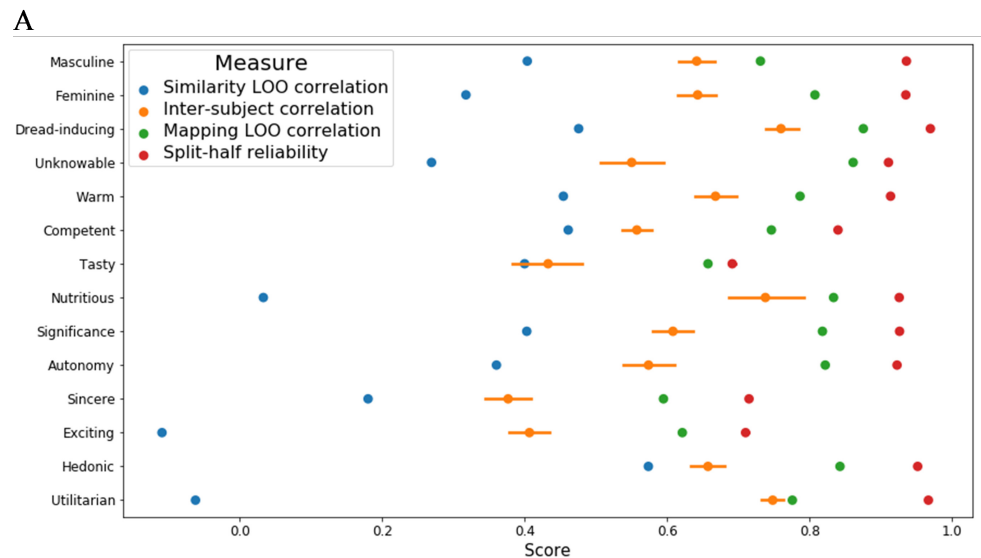

B

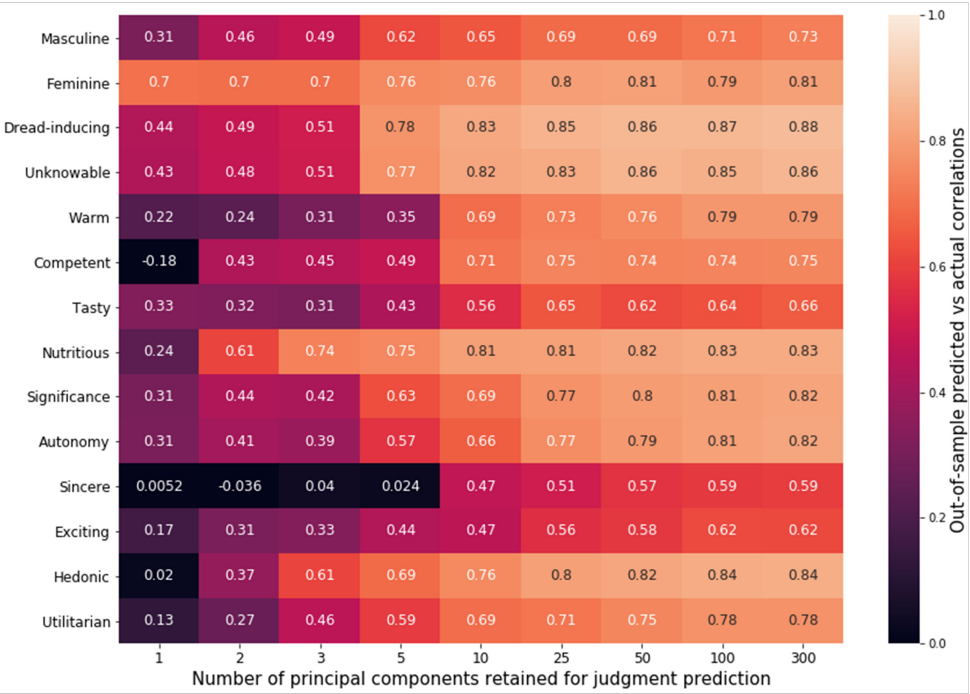

C

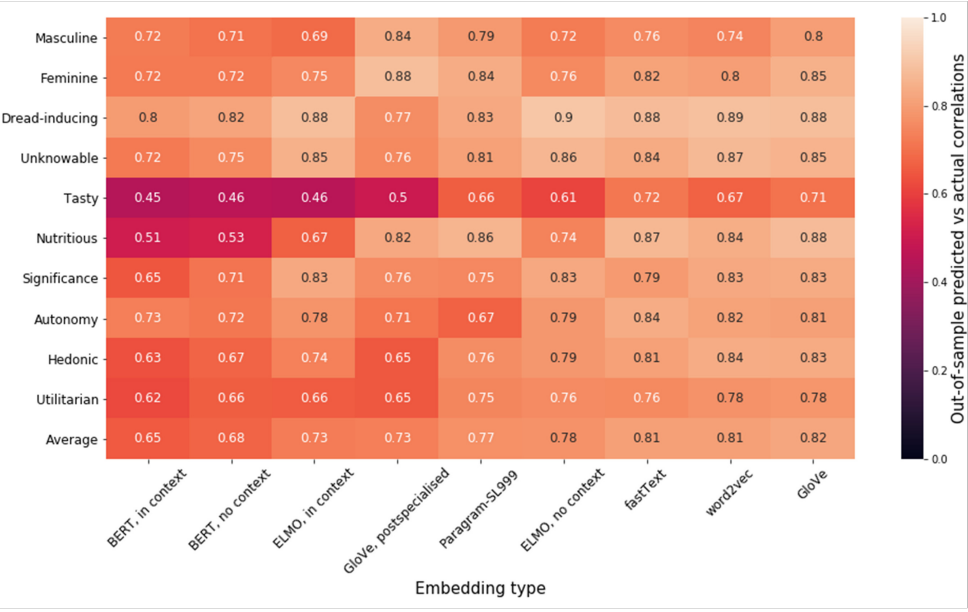

Figure 3: (A) Performance of the similarity baseline and mapping approach compared to inter-subject correlation (IS-r) and split-half reliability (error bars too small to visualize). The similarity baseline consistently performs under inter-subject correlation, while mapping outperforms this measure of reliability and approaches split-half reliability. $(B)$ Pearson correlations between predicted and actual judgments for every judgment dimension and varying numbers of retained principal components. Judgment domains (brands, goods, traits, etc.) can be compressed to 5 to 25 principal components while preserving judgment prediction accuracy. $(C)$ Pearson correlations between out-of-sample predicted and actual judgments for every judgment dimension except warmth and competence, for different word embedding models. 


\footnotetext{
${ }^{2}$ In principle, one could obtain, e.g., GloVe vectors for people's names and other MWE's just as in word2vec. All that is required is tokenizing the desired MWE's as individual "words" in the corpus before computing the global co-occurrence matrix for GloVe.
} 
dimensions, leading to their poorer overall accuracy. This leaves an ambiguous picture regarding the importance of using word embeddings that respect word-word similarity relations to model at least the present kinds of judgments (cf. the utility of similarity-specialised embeddings in other downstream NLP tasks like sentiment analysis [32] and lexical text simplification [33]). Aside from this, it is a bit difficult to interpret differences between the embedding models, given the conflation we noted before between training algorithm and training corpus. Our main point, however, is that our approach is robust to a range of training algorithms and corpora.

\subsection{Psychological Substrates of Judgment}

The ridge regression approach used in most of the above tests involves learning a (regularized) linear mapping from the semantic space to the judgment dimension. The best-fit weights for this mapping have the same dimensionality as the semantic space, and can thus be seen as representing a vector in this space. Judgment items whose vectors project strongly onto the weight vector (typically judgment items whose vectors are highly similar to the weight vector) will be predicted to have the highest judgment ratings. Given this interpretation, we can ask what other objects and concepts (that may not necessarily be judgment targets themselves) project strongly onto the weight vector. Intuitively, these would be the objects and concepts that are most related to the judgment, and may correspond to the judgment-relevant qualities that people evaluate when generating their responses. Uncovering these words and concepts would shed light on the psychological substrates of the judgment in consideration.

We used two methods to uncover these psychological substrates. Our first method involved obtaining word embedding representations for the 5,000 most common words in English that were not also judgment targets, and passing these embeddings through our trained ridge regression mapping to determine the associations of these words with our 14 judgment dimensions. We then computed the difference between a word's predicted association with one dimension (e.g., masculinity) and its predicted association with the complementary dimension (e.g., femininity), to find the words most strongly associated with one dimension relative to the other. Our second method used a similar approach, applied to word lexicons corresponding to 256 core psychological constructs from the General Inquirer (GI) and Linguistic Inquiry and Word Count (LIWC) dictionaries [36, 37]. In the SI we present additional details of these methods, as well as the results of these methods (see Figures S5 and S6 for summary visualizations, and Tables S3 and S4 for exhaustive reuslts). These results show, for example, that work-related interpersonal concerns are most associated with masculine traits whereas home and family concerns are most associated with feminine traits; passivity and submission are associated with high-warmth/low-competence individuals and strength and hostility are associated with high-competence/low-warmth individuals; natural objects are highly associated with nutrition; moral values and values of love and friendship are associated with sincere brands whereas values of power and respect are associated with exciting brands; and need-related motivations are associated with hedonic goods whereas means-related motivations are associated with utilitarian goods. These results conform with our intuition and with prior empirical work [38, 39, 40], despite not explicitly eliciting judgments about psychological constructs such as work-related or home-related interpersonal concerns and means-related or need-related motivations. Of course it is also possible to use these results to generate novel behavioral hypotheses, in a bottom up manner, which can be tested in subsequent empirical work.

\section{Discussion}

Despite the ubiquity of human judgment, until now we have had limited ability to predict arbitrary human judgments of objects and concepts, as capturing the rich knowledge used to make predictions has been difficult or impossible. Here we demonstrated in a pre-registered study that word embeddings - vector representations for words and concepts based on statistics of language use - proxy this knowledge and can predict 14 diverse judgments across the behavioral sciences with a high degree of accuracy, at both a group- and individual-level. We also showed that the learned mapping from word embeddings to judgments can also be used to explore the conceptual underpinnings of judgments, by mapping non-judgment target entities onto the judgment dimension.

We view the present approach as a modern extension to classical psychometric approaches used to uncover the underlying representations used for making judgments [15, 16, 17]. However, the present approach offers several advantages over classical techniques. First, the only human data that our approach requires is a (relatively) small number of judgment ratings to train a predictive model. Once a satisfactory model has been trained, no new human psychometric data is required to predict judgments for new entities. Second, word embeddings provide a single representational space to predict and understand behavior across many important domains, judgment target entities (objects, people, organizations, social relations, traits), and judgment dimensions. We believe that using a single representational space to model judgments relevant to different disciplines can provide cohesion to the behavioral sciences that traditionally rely on different methods and data. Third, word embeddings - owing to their high dimensionality - capture more 
knowledge about judgment targets than can realistically be collected from human participants, especially when the relevant knowledge used to make a particular judgment is not already theoretically well-understood and thus surveyed from human participants. Capturing a great degree of knowledge leads to the high predictive accuracy we have achieved here, which we suggest may be high enough for applications in downstream behavioral sciences and technologies.

We conclude by considering our work in the context of a recent meta-theoretical conversation in the social and behavioral sciences. As has been argued previously [41, 42], social, cognitive, and behavioral scientists have traditionally been focused on interpretable, explanatory models, to the detriment of developing models that make accurate out-of-sample predictions. Of course, this is undesirable to the extent that we think a good model requires external validity - having statistically significant, interpretable model coefficients is ultimately of limited use if a model can't predict new behavior with any accuracy. While some have claimed that behavioral scientists may sometimes need to choose between predictive or interpretable models [42], our models of judgment achieved unprecedented predictive accuracy, and substantial progress towards explanatory insights into judgments (sections Amount of Information Required for Prediction and Psychological Substrates of Judgment). We thus believe word embeddings are an excellent step towards fully predictive and interpretable models of the thousands of diverse and consequential judgments people make every day.

\section{Methods}

We recruited 354 participants (mean age $=31.89$ years, $46.19 \%$ female) through Prolific Academic. We limited our data collection to participants who were from the U.S. and had an approval rate above $80 \%$. Participants were only allowed to participate once, and they were paid $\$ 4.40$ each.

Using a between-subjects design, we randomly assigned each participant to one of the seven judgment domains - brands $(\mathrm{N}=54)$, consumer goods $(\mathrm{N}=51)$, traits $(\mathrm{N}=46)$, foods $(\mathrm{N}=55)$, occupations $(\mathrm{N}=49)$, risk sources $(\mathrm{N}=49)$, people $(\mathrm{N}=51)$. After being randomly assigned to one judgment domain, participants were instructed to rate 200 items on two dimensions from -100 (e.g. not at all significant) to 100 (e.g. extremely significant), one item at a time (see Figure S1 in the SI for scatterplots of judgments for all seven judgment domains). They were asked to check "not applicable" if they were not familiar with a particular item. There was a 30-second break after every 50 items, although participants were allowed to proceed without breaks if they so chose. Participants' age and gender information were collected at the end of the study. The mean completion time was 38 minutes; the median was 33 minutes.

In line with our pre-registered analysis plan, we excluded participants who took more than 1 hour to complete the study or indicated being unfamiliar with more than $25 \%$ of the items. Accordingly, one person was dropped from the occupation condition, one from risk, 11 from brands, 16 from people, and 36 from foods. An item was excluded if fewer than $25 \%$ of participants indicated being familiar with it. One brand, three people, and 38 foods were accordingly dropped. Retaining all items and participants does not affect our main result substantially: for a ridge regression with $\lambda$ set to 10, the mean Pearson correlation coefficient between predicted and actual judgments is .763 vs .769 under our pre-registered exclusion criteria. Taste and nutrition have the largest drop in performance, from $r=.66$ to $r=.59$ and $r=.83$ to $r=.79$, respectively, when retaining all items and participants.

Detailed instructions, survey questions and items, and exclusion criteria can be found at https://osf.io/t7qyb/

We adopt our baseline/similarity-based approach from [28]. This method works as follows: First, we select words reflective of high and low ends of some judgment dimension. For example, the occupation significance dimension was represented by the words significant, meaningful, important and insignificant, meaningless, unimportant, pointless. Where possible, we chose words used in previous literature to define the dimensions (see SI for these words). Then, for each judgment dimension, the average pairwise vector difference between each possible pair of high and low words is computed to obtain a single vector $d$ representing that dimension. Last, to obtain a score for a judgment target entity on that dimension, we compute the dot product between the target entity's embedding $x_{i}$ and the dimension embedding, $d * x_{i}$. As stated in Results, this method essentially computes the similarity of a judgment target to words high relative to words low along the dimension of interest.

Our mapping approach trains various supervised learning algorithms to predict judgments from word embeddings. More formally, we are training a model to predict a numerical rating $y_{i}$ for a judgment target $i$, from its 300 dimensional word embedding $x_{i}$, where $x_{i j}$ is the value of the judgment target on dimension $j$ of its embedding. Given that we have fewer judgment targets $(<=200)$ than independent variables (300), we chose three regularized regression techniques: lasso, ridge, and support vector machines. We also included k-nearest neighbors (KNN) regression since it was used in work on predicting risk perceptions with word embeddings [19]. We describe our secondary models (lasso, SVR, KNN) more in the SI, and instead focus here on our primary model, ridge regression. Ridge regression entails learning a vector of coefficients $\beta_{j}$ for the 300 dimensions of our embeddings, such that the following loss expression is minimized: 


$$
\sum_{i=1}^{n}\left(y_{i}-\sum_{j=1}^{p} x_{i j} \beta_{j}\right)^{2}+\lambda \sum_{j=1}^{p} \beta_{j}^{2}
$$

The left term of the loss expression is merely ordinary least squares regression. The additional sum on the right is a "penalty term" which pushes coefficients towards 0 , depending on the strength of the regularization parameter, $\lambda$. We searched for the optimal penalty strength by a cross-validation procedure described in the SI, and found $\lambda=10$ yielded the best out-of-sample r-squared and root mean squared error. Ridge with $\lambda=10$ is thus the model we use throughout the paper. (We performed a similar search for our other models' optimal hyperparameters). All our supervised models were implemented in the Scikit-Learn machine learning library for Python [43].

Notice that ridge (and lasso, or any other linear mapping) is like our similarity-based approach in that both produce a judgment rating by computing the dot product between a word embedding and a vector representing a judgment dimension. The difference between the approaches is that the similarity approach stipulates the vector for the judgment dimension, while the mapping approach learns the best vector given some true human judgments. While the similarity approach can score a judgment target on some dimension without any human data, these scores correlate with actual human judgments far worse than do predictions generated by the mapping approach. Whether one prefers accuracy or the lack of need for training data is likely to depend on one's subjective preferences or goals as a modeler [41]. Applied behavioral scientists, at least, likely want accurate predictions of human judgments so that they can predict important downstream cognition and behavior (see "Example Applications" of Table 1).

\section{Acknowledgments}

Thanks to members of the Computational Behavioral Science Lab and the Integrative Language Science and Technology seminar for their feedback on this work. This work was funded by National Science Foundation grant SES-1626825, and the Alfred P. Sloan Foundation.

\section{References}

[1] Alessandro Lenci. Distributional models of word meaning. Annual Review of Linguistics, 4:151-171, 2018.

[2] Peter D Turney and Patrick Pantel. From frequency to meaning: Vector space models of semantics. Journal of Artificial Intelligence Research, 37:141-188, 2010.

[3] Paweł Mandera, Emmanuel Keuleers, and Marc Brysbaert. Explaining human performance in psycholinguistic tasks with models of semantic similarity based on prediction and counting: A review and empirical validation. Journal of Memory and Language, 92:57-78, 2017.

[4] Elia Bruni, Nam-Khanh Tran, and Marco Baroni. Multimodal distributional semantics. Journal of Artificial Intelligence Research, 49:1-47, 2014.

[5] Felix Hill, Roi Reichart, and Anna Korhonen. Simlex-999: Evaluating semantic models with (genuine) similarity estimation. Computational Linguistics, 41(4):665-695, 2015.

[6] Markus J Hofmann, Chris Biemann, Chris Westbury, Mariam Murusidze, Markus Conrad, and Arthur M Jacobs. Simple co-occurrence statistics reproducibly predict association ratings. Cognitive Science, 42(7):2287-2312, 2018.

[7] Joseph Levy, John Bullinaria, and Samantha McCormick. Semantic vector evaluation and human performance on a new vocabulary MCQ test. In Proceedings of the 39th Annual Conference of the Cognitive Science Society, pages 2549-2554, 2017.

[8] Sudeep Bhatia. Associative judgment and vector space semantics. Psychological Review, 124(1):1-20, 2017.

[9] M Karl Healey and Michael J Kahana. A four-component model of age-related memory change. Psychological Review, 123(1):23-69, 2016.

[10] Thomas T Hills, Michael N Jones, and Peter M Todd. Optimal foraging in semantic memory. Psychological Review, 119(2):431-440, 2012.

[11] Michael N Jones, Walter Kintsch, and Douglas JK Mewhort. High-dimensional semantic space accounts of priming. Journal of Memory and Language, 55(4):534-552, 2006.

[12] Sudeep Bhatia. Semantic processes in preferential decision making. Journal of Experimental Psychology. Learning, Memory, and Cognition, 2018. 
[13] Aylin Caliskan, Joanna J Bryson, and Arvind Narayanan. Semantics derived automatically from language corpora contain human-like biases. Science, 356(6334):183-186, 2017.

[14] Nikhil Garg, Londa Schiebinger, Dan Jurafsky, and James Zou. Word embeddings quantify 100 years of gender and ethnic stereotypes. Proceedings of the National Academy of Sciences, 115(16):E3635-E3644, 2018.

[15] Roger N Shepard. Multidimensional scaling, tree-fitting, and clustering. Science, 210(4468):390-398, 1980.

[16] Ken McRae, George S Cree, Mark S Seidenberg, and Chris McNorgan. Semantic feature production norms for a large set of living and nonliving things. Behavior Research Methods, 37(4):547-559, 2005.

[17] Paul Slovic. Perception of risk. Science, 236(4799):280-285, 1987.

[18] Geoff Hollis, Chris Westbury, and Lianne Lefsrud. Extrapolating human judgments from skip-gram vector representations of word meaning. The Quarterly Journal of Experimental Psychology, 70(8):1603-1619, 2017.

[19] Sudeep Bhatia. Predicting risk perception: New insights from data science. Management Science, in press.

[20] Tomas Mikolov, Ilya Sutskever, Kai Chen, Greg S Corrado, and Jeff Dean. Distributed representations of words and phrases and their compositionality. In Advances in Neural Information Processing Systems, pages 3111-3119, 2013.

[21] Sandra L Bem. The measurement of psychological androgyny. Journal of Consulting and Clinical Psychology, 42(2):155-162, 1974.

[22] Seymour Rosenberg, Carnot Nelson, and PS Vivekananthan. A multidimensional approach to the structure of personality impressions. Journal of Personality and Social Psychology, 9(4):283-294, 1968.

[23] Amy JC Cuddy, Susan T Fiske, Peter Glick, and Jun Xu. A model of (often mixed) stereotype content: Competence and warmth respectively follow from perceived status and competition. Journal of Personality and Social Psychology, 82(6):878-902, 2002.

[24] Rajagopal Raghunathan, Rebecca Walker Naylor, and Wayne D Hoyer. The unhealthy= tasty intuition and its effects on taste inferences, enjoyment, and choice of food products. Journal of Marketing, 70(4):170-184, 2006.

[25] J Richard Hackman and Greg R Oldham. Motivation through the design of work: Test of a theory. Organizational Behavior and Human Performance, 16(2):250-279, 1976.

[26] Jennifer L Aaker. Dimensions of brand personality. Journal of Marketing Research, pages 347-356, 1997.

[27] R Batra and OT Ahtola. Sources of the hedonic and utilitarian measuring attitudes consumer. Consumer Attitudes, 2(2):159-170, 1990.

[28] Gabriel Grand, Idan Asher Blank, Francisco Pereira, and Evelina Fedorenko. Semantic projection: recovering human knowledge of multiple, distinct object features from word embeddings. arXiv preprint arXiv:1802.01241, 2018.

[29] Mohammad Taher Pilehvar and José Camacho-Collados. Wic: 10, 000 example pairs for evaluating contextsensitive representations. CoRR, abs/1808.09121, 2018.

[30] Jeffrey Pennington, Richard Socher, and Christopher Manning. Glove: Global vectors for word representation. In Proceedings of the 2014 Conference on Empirical Methods in Natural Language Processing (EMNLP), pages 1532-1543, 2014.

[31] Tomas Mikolov, Edouard Grave, Piotr Bojanowski, Christian Puhrsch, and Armand Joulin. Advances in pretraining distributed word representations. In Proceedings of the International Conference on Language Resources and Evaluation (LREC 2018), 2018.

[32] John Wieting, Mohit Bansal, Kevin Gimpel, Karen Livescu, and Dan Roth. From paraphrase database to compositional paraphrase model and back. arXiv preprint arXiv:1506.03487, 2015.

[33] Edoardo Maria Ponti, Ivan Vulic, Goran Glavas, Nikola Mrksic, and Anna Korhonen. Adversarial propagation and zero-shot cross-lingual transfer of word vector specialization. CoRR, abs/1809.04163, 2018.

[34] Matthew E Peters, Mark Neumann, Mohit Iyyer, Matt Gardner, Christopher Clark, Kenton Lee, and Luke Zettlemoyer. Deep contextualized word representations. arXiv preprint arXiv:1802.05365, 2018.

[35] Jacob Devlin, Ming-Wei Chang, Kenton Lee, and Kristina Toutanova. Bert: Pre-training of deep bidirectional transformers for language understanding. arXiv preprint arXiv:1810.04805, 2018.

[36] Philip J Stone, Dexter C Dunphy, and Marshall S Smith. The general inquirer: A computer approach to content analysis. 1966.

[37] James W Pennebaker, Ryan L Boyd, Kayla Jordan, and Kate Blackburn. The development and psychometric properties of liwc2015. Technical report, 2015. 
[38] Amy JC Cuddy, Susan T Fiske, and Peter Glick. Warmth and competence as universal dimensions of social perception: The stereotype content model and the bias map. Advances in experimental social psychology, 40:61-149, 2008.

[39] Madeline E Heilman. Gender stereotypes and workplace bias. Research in organizational Behavior, 32:113-135, 2012.

[40] Paul Rozin, Mark Spranca, Zeev Krieger, Ruth Neuhaus, Darlene Surillo, Amy Swerdlin, and Katherine Wood. Preference for natural: instrumental and ideational/moral motivations, and the contrast between foods and medicines. Appetite, 43(2):147-154, 2004.

[41] Jake M Hofman, Amit Sharma, and Duncan J Watts. Prediction and explanation in social systems. Science, 355(6324):486-488, 2017.

[42] Tal Yarkoni and Jacob Westfall. Choosing prediction over explanation in psychology: Lessons from machine learning. Perspectives on Psychological Science, 12(6):1100-1122, 2017.

[43] Fabian Pedregosa, Gaël Varoquaux, Alexandre Gramfort, Vincent Michel, Bertrand Thirion, Olivier Grisel, Mathieu Blondel, Peter Prettenhofer, Ron Weiss, Vincent Dubourg, et al. Scikit-learn: Machine learning in Python. Journal of Machine Learning Research, 12(Oct):2825-2830, 2011. 